\title{
Qualidade de vida de mulheres climatéricas comparada com o uso de medicamentos
}

\author{
Quality of life in menopausa/ women versus use of medicines
}

Recebido em: 22/04/2015 Aceito em: $\quad 02 / 06 / 2015$
Ronilson Ferreira FREITAS ${ }^{1}$, Tahiana Ferreira FREITAS ${ }^{2}$, Thércia Guedes

VIANA ${ }^{3}$, Vanessa de Andrade ROYO ${ }^{4}$, Josiane Santos Brant ROCHA 4 , Vivianne Margareth Chaves Pereira REIS ${ }^{4}$, Jadson Rabelo ASSIS ${ }^{2}$, Dorothéa Schmidt FRANÇA ${ }^{5}$

${ }^{1}$ Universidade Federal dos Vales do Jequitinhonha e Mucuri - UFVJM. Campus JK - Rodovia MGT 367 $\mathrm{Km}$ 583, $n^{\circ}$ 5000. Alto da Jacuba, CEP 39100-000, Diamantina, MG, Brasil. ${ }^{2}$ Faculdade de Saúde Ibituruna, Faculdades Integradas Norte de Minas - FASI/FUNORTE. Avenida Nice, $n^{\circ}$ 99, Ibituruna, CEP 39401-303. Montes Claros, MG, Brasil. ${ }^{3}$ Universidade Federal de Minas Gerais - UFMG. Avenida Presidente Antônio Carlos, 6627 - Pampulha, CEP 31270-901, Belo Horizonte, MG, Brasil. ${ }^{4}$ Universidade Estadual de Montes

Claros - Unimotes. Endereço: Avenida Rui Braga - Vila Mauriceia, CEP 39401-089, Montes Claros, MG, Brasil. ${ }^{5}$ Faculdades Integradas Pitágoras de Montes Claros - FIPMoc. Avenida Rui Braga, Vila Mauriceia, CEP 39401-089, Montes Claros, MG, Brasil.E-mail: ronnypharma@bol.com.br

\section{ABSTRACT}

Brazil is passing through an aging process, with a significant increase in the number of women experiencing the climacteric syndrome and medicines use. Thus, the objective was to compare the quality of life of menopausal women in use or not of drugs. We conducted a descriptive, cross-sectional, quantitative nature, involving 626 menopausal women seen at ESF Montes Claros, MG. Data were collected related to sociodemographic and clinical profile of the menopausal women. Menopause Rating Scale (MRS) was used to evaluate the quality of life. To compare the quality of life with the use of medications, we used the Student $t$ test, with $5 \%$ significance level. The results showed predominance of women aged between 45-59 years (59.3\%), with less than nine years of formal education $(72.0$ $\%)$, with a steady partner (64.2\%), and one and two minimum wages income (43.9\%). Concerning physical activity, $77.8 \%$ of them did not practice physical activity. Non-smoking women were the most prevalent $(83.7 \%)$. Among the respondents, $75.2 \%$ reported being hypertensive and $70.8 \%$ of women reported using drugs in general in their day to day. Comparing life quality with drug use revealed differences $(p<0.05)$ in the total score and somatic- vegetative symptoms, psychological and urogenital, with steeper average values in women who used drugs.

Keywords: menopause; wellfare; life quality; use of medicines

\section{RESUMO}

O Brasil está em um processo de envelhecimento, com aumento significativo do número de mulheres que vivenciam a síndrome do climáterio e o consumo de medicamentos. Assim, foi realizado um estudo descritivo, do tipo transversal, de cunho quantitativo, envolvendo 626 mulheres climatéricas atendidas nas ESF de Montes Claros, MG. Foram coletados dados relacionados ao perfil sócio-demográfico e clínico das mulheres climatéricas. Para a avaliação da qualidade de vida, foi utilizada a Escala de Avaliação da Menopausa (Menopause Rating Scale - MRS). Para comparar a qualidade de vida com o uso de medicamentos, foi utilizado o teste $t$ de student, com nível de significância de $5 \%$. Houve predominância de mulheres de faixa etária entre 45 a 59 anos (59,3\%) com grau de instrução menor que nove anos de estudo formal $(72,0 \%)$, com companheiro fixo $(64,2 \%)$ e renda familiar entre um e dois salários mínimos $(43,9 \%)$. No que se refere à prática de atividade física, $77,8 \%$ das mulheres responderam não praticar atividade física. Houve prevalência de mulheres não fumantes $(83,7 \%)$. Dentre as entrevistadas, $75,2 \%$ informaram ser hipertensas e 70,8\% informaram utilizar medicamentos de maneira geral no seu dia a dia. A comparação dos itens da qualidade de vida com o consumo de medicamentos revelou a presença de diferenças $(\mathrm{p}<0,05)$ no escore total da escala e em relação aos sintomas somato-vegetativos, psicológicos e urogenitais, com valores médios mais acentuados nas mulheres que utilizavam medicamentos.

Palavras chave: climatério; qualidade de vida; uso de medicamentos. 


\section{INTRODUÇÃO}

O Brasil tem vivenciado um processo de envelhecimento demográfico com particularidades que o destacam na escala mundial (1). Com isso, tem sido observado um aumento significativo do número de mulheres que atingem o climatério (2). O climatério corresponde à fase da vida das mulheres no qual ocorre a transição do período reprodutivo para o não reprodutivo. Considera-se que inicia por volta dos 40 anos e termine na senescência, ou seja, em torno dos 65 anos (3). Nessa fase, ocorrem alterações endócrinas devido ao declínio da atividade ovariana, às mudanças biológicas em função da diminuição da fertilidade e às mudanças clínicas consequentes das alterações do ciclo menstrual e de uma variedade de sintomas (4).

A diminuição de estrogênio circulante na perimenopausa é um processo que ocasiona sintomas desconfortáveis e que afetam o bem-estar da mulher, favorecendo o desenvolvimento dos sintomas vasomotores, psicológicos e urogenitais. Em decorrência do hipoestrogenismo, são observados: ondas de calor, sudorese noturna, secura vaginal, enfraquecimento da musculatura do assoalho pélvico, dispareunia, insônia, alterações de humor e depressão (5).

Diante dessa realidade, é necessária uma maior atenção dos serviços de saúde, em especial no que se refere à síndrome do climatério, que compreende um conjunto de sinais e sintomas que prejudicam a sensação de bem-estar das mulheres, durante essa fase da vida (6). Várias são as possibilidades de intervenção no climatério, cuja efetividade depende de uma escuta qualificada dessas mulheres, das questões ocultas em suas queixas, dos seus sentimentos e percepções acerca do seu envelhecimento (7). Nesse contexto, admite-se que o uso de medicamentos constitui uma intervenção importante para a recuperação e manutenção da saúde de grande parcela das mulheres no climatério (1). Para tanto, é indispensável que a mulher climatérica tenha espaço para expressar os seus sentimentos acerca do momento que está vivendo e as dificuldades que está sentindo, recebendo informações sobre as mudanças que o seu corpo está sofrendo e as suas implicações para a sua saúde. É importante que os profissionais de saúde acolham adequadamente as mulheres climatéricas, permitindo que exponham as suas dúvidas e receios (7).

Nessa conjuntura, é reconhecida a importância de saber monitorar o consumo dos medicamentos utilizados para aliviar os sintomas advindos do climatério, preservando o bem-estar e a qualidade de vida dessa população (8). Entretanto, a carência de políticas públicas efe- tivas na região do Norte de Minas Gerais direcionadas a essa clientela, salienta a relevância de estudos nessa área, capazes de contribuir para a transformação social e melhorar a asistência do profissional farmacêutico para essa clientela.

Dessa forma, o presente estudo foi realizado com o objetivo de comparar a qualidade de vida de mulheres climatéricas em uso ou não de medicamentos.

\section{MÉTODO}

O presente estudo caracterizou-se por ser descritivo, do tipo transversal, de cunho quantitativo. Os critérios de inclusão compreenderam 626 pacientes do sexo feminino, com idade entre 40 e 65 anos, que não faziam uso de terapia de reposição hormonal e se encontravam aguardando atendimento médico nas Estratégias de Saúde da Família (ESF) da cidade de Montes Claros, Norte do Estado de Minas Gerais, Brasil. Todas as mulheres foram informadas sobre os procedimentos e suas implicações, e confirmaram a sua participação assinando o Termo de Consentimento Livre e Esclarecido.

Entre os dados coletados na avaliação sócio-demográfica estavam: faixa etária ( 40 a 44 anos, 45 a 59 anos e 60 a 65 anos), grau de instrução (menos de nove anos de estudo formal e mais de nove anos de estudo formal), estado civil (com companheiro fixo e sem companheiro fixo) e renda familiar (menos de um salário mínimo, entre um e dois salários mínimos e três ou mais salários mínimos). Para a avaliação clínica, as váriaveis utilizadas foram: atividade física (prática e não prática), tabagismo (fumante e não fumante), hipertensão (hipertensa e não hipertensa) e medicamentos (utiliza e não utiliza medicamentos).

Para a avaliação da qualidade de vida foi utilizada a Escala de Avaliação da Menopausa (Menopause Rating Scale - MRS) (9), desenvolvida na Alemanha, tendo sido validada para a língua portuguesa no Brasil. Possui onze questões avaliadas numa escala de zero (ausência de sintomas) até quatro (maior severidade) e distribuídas em três domínios: sintomas somato-vegetativos, sintomas urogenitais e sintomas psicológicos. O escore total do MRS é obtido por meio do somatório da pontuação de cada domínio, de forma que, quanto maior for esta, mais severa a sintomatologia e pior a qualidade de vida. A intensidade geral da sintomatologia climatérica referida foi categorizada segundo a severidade dos sintomas que compõe cada domínio do MRS em: sintomatologia ausente ou ocasional ( $0-4$ pontos), leve (5-8 pontos), moderada ( $9-15$ pontos) ou severa ( $>16$ pontos) (10). 
Os dados foram analisados com o programa SPSS (versão 20.0). Inicialmente, os dados foram descritos utilizando-se estatística descritiva das variáveis sócio-demográficas e clínicas. Para comparar a qualidade de vida com o uso de medicamentos, foi utilizado o teste $t$ de student. Em todas as análises realizadas, aceitou-se um nível de significância de 5\%.

A pesquisa foi submetida ao Comitê de Ética em Pesquisa da Universidade Estadual de Montes Claros Unimontes, tendo sido aprovado sob o parecer de número $311.628 / 2013$.

\section{RESULTADOS}

A distribuição das características sociodemográficas é apresentada na Tabela 1. Houve predominância de mulheres de faixa etária entre 45 a 59 anos (59,3\%), com grau de instrução com menos de nove anos de estudo formal $(72,0 \%)$, estado civil com companheiro fixo $(64,2 \%)$ e renda familiar entre um e dois salários mínimos (43,9\%).

Tabela 1 - Análise descritiva sócio-demográfica de mulheres climatéricas atendidas na Estatégia de Saúde da Família em Montes Claros, MG (2013).

\begin{tabular}{ccc|}
\hline Perfil Sociodemográfico & Váriáveis & $(\%)(\mathbf{n})$ \\
\hline \multirow{2}{*}{ Faixa Etária } & 40 a 44 anos & $17,7-111$ \\
& 45 a 59 anos & $59,3-371$ \\
Grau de Instrução & 60 a 65 anos & $23,0-144$ \\
& Menos de 9 anos de estudo formal & $72,0-451$ \\
Estado Civil & Mais de 9 anos de estudo formal & $28,0-175$ \\
& Companheiro fixo & $64,2-402$ \\
Renda Familiar & Sem companheiro fixo & $35,8-224$ \\
& Menos de 1 salário mínimo & $42,3-265$ \\
& Entre 1 e 2 salários mínimos & $43,9-275$ \\
\end{tabular}

(\%) - Porcentagem observada; ( $n$ ) - Tamanho da Amostra.

Quanto às variáveis clínicas, os dados estão disponíveis na tabela 2 . No que se refere à prática de atividade física, $77,8 \%$ da mulheres responderam não praticar atividade física.

Tabela 2 - Análise descritiva clínica de mulheres climatéricas atendidas na Estatégia de Saúde da Família em Montes Claros, MG (2013).

\begin{tabular}{|c|c|c|}
\hline Perfil Clínico & Váriáveis & $\%(\mathbf{n})$ \\
\hline \multirow{2}{*}{ Atividade Física } & Pratica & $22,2(139)$ \\
& Não pratica & $77,8(487)$ \\
\hline \multirow{2}{*}{ Tabagismo } & Fumante & $16,3(102)$ \\
\hline \multirow{2}{*}{ Hipertensão } & Não fumante & $83,7(524)$ \\
\hline \multirow{2}{*}{ Medicamentos } & Hipertensa & $75,2(471)$ \\
& Não hipertensa & $24,8(155)$ \\
\hline
\end{tabular}

(\%) - Porcentagem observada; ( $\mathrm{n}$ - Tamanho da Amostra.

Houve prevalência de mulheres não fumantes $(83,7 \%)$. Entre as entrevistadas, $75,2 \%$ informaram ser hipertensas e $70,8 \%$ das mulheres informaram utilizar medicamentos de maneira geral no seu dia a dia.

A comparação dos itens da qualidade de vida com o consumo de medicamentos (Tabela 3) revelou a presença de diferenças $(p<0,05)$ no escore total da escala. Em relação aos sintomas somatovegetativos, psicológicos e urogenitais, com valores médios mais acentuados nas mulheres que utilizavam medicamentos, foi revelada a presença de uma sintomatologia leve para os dois grupos. Em relação ao escore total da escala, a sintomatologia moderada foi também a que assumiu maior dimensão.

\section{DISCUSSÃO}

Atualmente tem sido observado um aumento significativo na população climatérica em decorrência da melhoria na assistência social, médica e no controle e tratamento das doenças relacionadas a essa fase da vida. Entretanto, ainda é notável o impacto negativo da qualidade de vida das mulheres em decorrência do estado menopausal (11). 
Tabela 3 - Qualidade de vida de mulheres climátericas atendidas na Estatégia de Saúde da Família em Montes Claros, MG (2013) versus o uso de medicamentos.

\begin{tabular}{|c|c|c|}
\hline Qualidade de Vida/sintomas & $\begin{array}{c}\text { Utiliza Medicamentos } \\
(\mathrm{n}=443)\end{array}$ & $\begin{array}{c}\text { Não Utiliza Medicamentos } \\
(\mathrm{n}=183)\end{array}$ \\
\hline somato-vegetativos & $6,6 \pm 3,8$ & $4,4 \pm 3,8^{*}$ \\
\hline psicológicos & $6,8 \pm 4,3$ & $5,2 \pm 4,5^{*}$ \\
\hline urogenitais & $2,4 \pm 2,6$ & $1,5 \pm 2,2^{*}$ \\
\hline Escore total & $15,8 \pm 8,6$ & $11,2 \pm 8,8^{*}$ \\
\hline
\end{tabular}

$\mathrm{DP}=$ desvio padrão; $\mathrm{n}=$ número de elementos da amostra; $\mathrm{p}<0,05$

Um dos aspectos que tem contribuído para uma regressão nos níveis da qualidade de vida das mulheres no climatério é a falta de atividade física. Estudos tem mostrado o efeito benéfico do exercício na prevenção primária e secundária de diversas doenças, como a hipertensão arterial, cardiopatia isquêmica, diabetes, osteoporose, entre outras. São relatados também efeitos benéficos do exercício sobre os fogachos e a depressão psíquica no climatério. O exerício físico preserva ainda a massa óssea, tanto por ação direta do impacto sobre o esqueleto, como por ação indireta, pelo aumento da força muscular (12).

No que tange ao tabagismo, esse estudo revelou que $16,3 \%(n=102)$ eram fumantes, um número significativo, uma vez que o hábito de fumar está associado com os riscos para o aparecimento de sintomas como calorões, possivelmente pelo efeito do metabolismo estrogênico ou dos efeitos termogênicos da nicotina (13), além dos problemas relacionados a doenças bronco-pulmonares, câncer de pulmão e elevado risco para desenvolvimento de doenças cardiovasculares (7).

Quanto ao desenvolvimento da hipertensão arterial sistêmica, o envelhecimento propicia alterações estruturais e funcionais no sistema cardiovascular que predispõe essa patologia, sendo que no estudo realizado foi possível observar que $75,2 \%(n=471)$ da amostra era hipertensa. Segundo Esperandio et al. (2013), nas mulheres, a partir dos 45 anos, as alterações próprias do climatério conferem aumento da pressão arterial e do risco cardiovascular(14). Para Coylewright et al. (2008), a menopausa, associada ao aumento da rigidez arterial devido ao avanço da idade, pode ser responsável pela elevada prevalência de hipertensão em idosas (15).

Nesse estudo, ainda foi possível observar que $70,8 \%$ (443) utilizavam medicamentos gerais no seu dia a dia. Segundo Gewehr et al. (2014), esse resultado é possível devido a grande incidência de cardiovasculares, osteoporose, hipotireoidismo, obesidade, diabetes mellitus e transtornos psicossociais que acometem as mulheres nesse período da vida (16), o que aumenta o consumo de medicamentos e, assim, as chances de eventos adversos relacionados ao uso de medicamentos, como as interações medicamentosas (17).

Com este estudo foi buscada a comparação entre a qualidade de vida de mulheres climatéricas e o consumo de medicamentos de maneira geral, procurando responder a algumas lacunas identificadas na literatura causadas pelo restrito número de estudos que avaliam a qualidade de vida de mulheres climatéricas (18). Segundo Arrais et al. (2005), o medicamento é um bem essencial à saúde e uma importante ferramenta terapêutica nas mãos dos médicos, sendo responsável por parte significativa da melhoria da qualidade e expectativa de vida da população (19). Entretanto, este resultado contradiz com os encontrados neste estudo, em que foi possível observar que mulheres que utilizam medicamentos apresentaram piores escores para a qualidade de vida. Esse fato pode ser justificado devido ao uso irracional e suas consequências, sendo que esse uso irracional pode elevar os problemas de saúde, bem como redução da qualidade de vida, além de aumentar os gastos na área da saúde, o que torna o tema de grande relevância para os que trabalham com saúde pública (19).

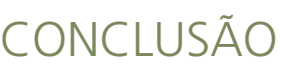

As mulheres climatéricas participantes deste estudo destacaram-se no consumo de medicamentos, sendo que, quando comparada a qualidade de vida com o uso de medicamentos de maneira geral, aquelas que utilizavam medicamentos apresentaram piores escores na qualidade de vida. Assim, urge uma melhor assistência à mulher no climatério, além do desenvolvimento de ações educativas voltadas para o uso racional de medicamentos nessa clientela. 
